\title{
Adding Consolidation Capecitabine to Neoadjuvant Chemoradiotherapy for Locally Advanced Rectal Cancer: A Propensity-Matched Comparative Study
}

\author{
Yifang Fang ${ }^{1 \dagger}$, Chengmin Sheng ${ }^{2 \dagger}$, Feng Ding ${ }^{1 \dagger}$, Weijie Zhao ${ }^{1 \dagger}$, Guoxian Guan ${ }^{1 *}$ and \\ Xing Liu ${ }^{1 *}$
}

${ }^{1}$ Department of Colorectal Surgery, The First Affiliated Hospital of Fujian Medical University, Fuzhou, China, ${ }^{2}$ Fuzhou Medical College of Nanchang University, Fuzhou, China

\section{OPEN ACCESS}

Edited by:

Qi Liu,

Fudan University, China

Reviewed by:

Takahiro Hiratsuka,

Oita University, Japan

Cihangir Akyol,

Ankara University, Turkey Mohammad Mohammadianpanah,

Shiraz University of Medical

Sciences, Iran

*Correspondence:

Xing Liu

fjmufylx@163.com

Guoxian Guan

fjxhggx@163.com

†These authors have contributed equally to this work

Specialty section: This article was submitted to

Surgical Oncology,

a section of the journal

Frontiers in Surgery

Received: 04 September 2021 Accepted: 27 December 2021

Published: 27 January 2022

Citation:

Fang Y, Sheng C, Ding F, Zhao W, Guan G and Liu X (2022) Adding

Consolidation Capecitabine to Neoadjuvant Chemoradiotherapy for Locally Advanced Rectal Cancer: A

Propensity-Matched Comparative

Study. Front. Surg. 8:770767.

doi: 10.3389/fsurg.2021.770767
Aim: To determine whether adding consolidation capecitabine chemotherapy without lengthening the waiting period influences pathological complete response (pCR) and short-term outcome of locally advanced rectal cancer (LARC) receiving neoadjuvant chemoradiotherapy (NCRT).

Method: Totally, 545 LARC who received NCRT and radical resection between 2010 and 2018 were enrolled. Short-term outcome and pCR rate were compared between patients with and without additional consolidation capecitabine. Logistic analysis was performed to identify predictors of pCR.

Results: After propensity score matching, 229 patients were matched in both NCRT and NCRT-Cape groups. Postoperative morbidity was comparable between groups except for operation time, which is lower in the NCRT group (213.2 \pm 67.4 vs. $227.9 \pm 70.5$, $p=0.025)$. Two groups achieved similar pCR rates ( $21.8 \mathrm{vs.} 22.7 \%, p=1.000)$. Tumor size $(\mathrm{OR}=0.439, p<0.001)$, time interval between NCRT and surgery $(\mathrm{OR}=1.241$, $p=0.003$ ), and post-NCRT carcinoembryonic antigen (OR $=0.880, p=0.008$ ) were significantly correlated with $\mathrm{PCR}$ in patients with $L A R C$. A predictive nomogram was constructed with a C-index of 0.787 and 0.741 on internal and external validation.

Conclusion: Adding consolidation capecitabine chemotherapy without lengthening CRT-to-surgery interval in LARC patients after NCRT does not seem to impact pCR or short-term outcome. A predictive nomogram for pCR was successful, and it could support treatment decision-making.

Keywords: rectal neoplasm, neoadjuvant chemoradiotherapy, capecitabine, prognosis, propensity score matched analysis

\section{INTRODUCTION}

Neoadjuvant chemoradiotherapy (NCRT) and radical surgery have become the standard treatment for locally advanced rectal cancer (LARC) (1). The benefits of this multimodal treatment have been well-documented, namely, tumor downsizing and downstaging, increased radical resection rate, and better local tumor control (2-4). Approximately $10-30 \%$ of LARC patients following NCRT 


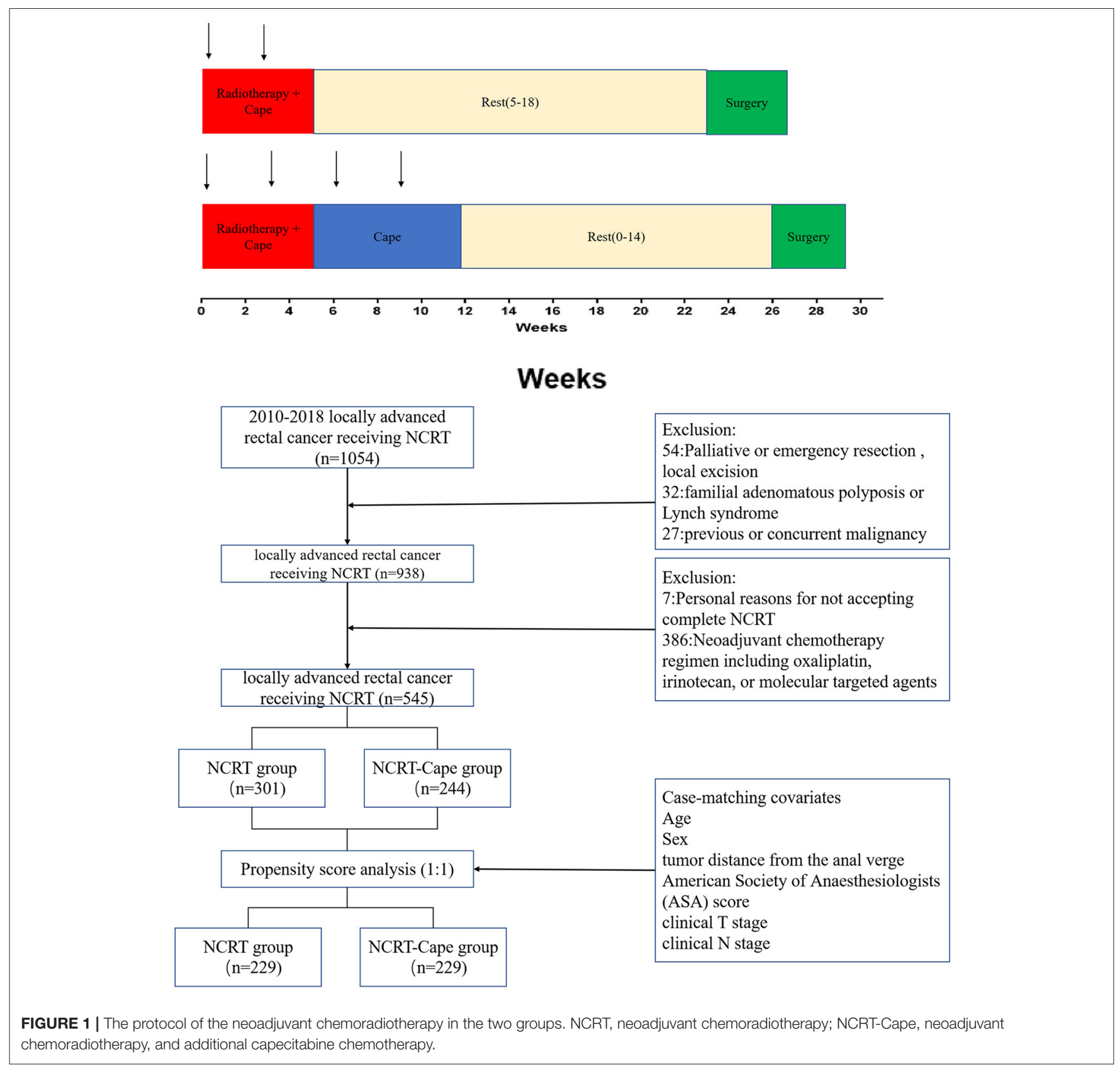

will develop a pathological complete response (pCR), together with a low recurrence rate (5-7).

Given the superior oncological outcome, organ preservation with a "watch and wait" strategy or local excision, has been proposed to patients achieving $\mathrm{PCR}$ to improve the quality of life and anal sphincter preserving rate. Consequently, increasing $\mathrm{pCR}$ rate has become a primary endpoint of clinical trials, which might increase patients with LARC who could potentially benefit from organ-preservation strategies. Many strategies have been adopted to maximize the $\mathrm{PCR}$ rate, namely, dose-escalated radiation (8), intensified neoadjuvant treatment [induction (9) or consolidation chemotherapy
$(1,8,10-16)]$, and lengthening the CRT-to-surgery interval (17).

Standard NCRT protocol using a continuous infusional 5fluorouracil $(5-\mathrm{Fu})$ for radiation sensitization has been shown to achieve tumor downstaging, but no improved overall survival. Growing evidence has demonstrated that the addition of oxaliplatin to NRT acquires equivalent oncological outcomes when compared to fluoropyrimidine-based CRT, but increases toxicities and cost (18-22). The inconvenience of using an intravenous continuous infusion of 5-FU resulted in the development of an oral fluoropyrimidine, capecitabine. A metaanalysis (23) has demonstrated equivalent efficacy of capecitabine 
TABLE 1 | Patient characteristics in patients with LARC after NCRT.

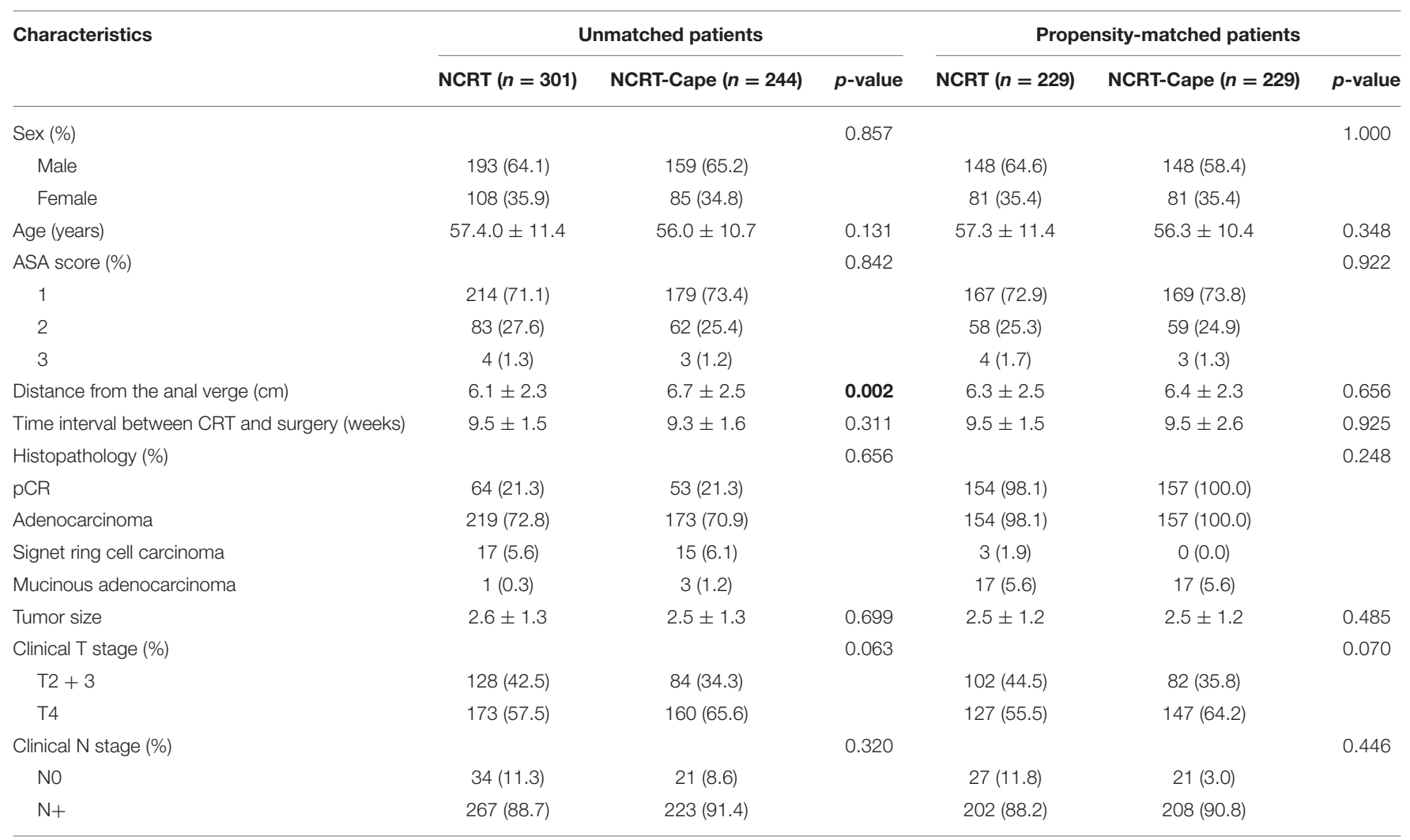

LARC, locally advanced rectal cancer; NCRT, neoadjuvant chemoradiotherapy; NCRT-Cape, neoadjuvant chemoradiotherapy and additional capecitabine chemotherapy; ASA, American society of anesthesiologists; CRT, chemoradiotherapy. Bold values indicate of statistical significance.

and infusional 5 -Fu in the neoadjuvant setting, suggesting capecitabine to be an alternative to 5-Fu-based CRT for LARC.

In this article, we aimed to determine whether adding 2 cycles of consolidation capecitabine without lengthening CRTto-surgery interval influences pCR rate and short-term outcome of LARC patients after NCRT. In addition, we sought to identify post-CRT determinants for $\mathrm{pCR}$, and to construct a nomogram that might be helpful during organ preservation strategy decision-making.

\section{PATIENTS AND METHOD}

\section{Patient Eligibility}

We performed a retrospective study based on propensity score matching. Between October 2010 and January 2018, patients with LARC who underwent curative resection and received capecitabine-based NCRT from our database. Patient inclusion criteria were: (1) clinical stage II or III (cT3/4 or cN1/2) disease; (2) pathologically proven rectal adenocarcinomas; and (3) tumors distance $<12 \mathrm{~cm}$ from the anal verge. Exclusion criteria included: (1) neoadjuvant chemotherapy regimen, namely, oxaliplatin, irinotecan, or molecular targeted agents; (2) previous or concurrent malignancy; (3) emergency or palliative resection; and (4) transanal local excision. This study was approved by the
Institutional Review Board of Fujian Medical University Union Hospital (2013051).

\section{Treatment Protocol and Follow-Up}

Patient assessments were performed at baseline for tumor staging using a digital rectal examination, colonoscopy, chest radiography, abdominopelvic MRI, and/or transrectal ultrasound. Preoperative radiotherapy consisted of $45 \mathrm{~Gy}$ to the pelvis for 5 weeks ( $180 \mathrm{cGy} / 25$ fractions) and a tumor boost of $5.4 \mathrm{~Gy}$. Concomitant chemotherapy was administered with oral capecitabine $\left(825 \mathrm{mg} / \mathrm{m}^{2}\right.$ two times daily from day 1 to day 14 per cycle, a total of two cycles during the preoperative radiotherapy). The treatment decision whether or not to add two-cycle consolidation capecitabine chemotherapy to NCRT was based on the disease stage. Surgery was carried out 6-20 weeks after the completion of radiation. Surgical techniques, namely, total mesorectal excision and high ligation of the inferior mesenteric artery, were routinely performed. After 3-4 weeks from surgery, adjuvant chemotherapy (FOLFOX or CapeOX) was considered for patients for 6 months. The treatment schema of our study is presented in Figure 1.

Patients were followed four times in the first 3 years, then twice for the next 2 years, and annually thereafter. Patient follow-up lasted until death or the cut-off date of October 31, 2018. 
TABLE 2 | Operative and post-operative outcomes in patients with LARC after NCRT.

\begin{tabular}{|c|c|c|c|}
\hline Characteristics & $\begin{array}{c}\text { NCRT }(n= \\
229)\end{array}$ & $\begin{array}{c}\text { NCRT-Cape } \\
(n=229)\end{array}$ & $p$-value \\
\hline Operative time (min) & $213.2 \pm 67.4$ & $227.9 \pm 70.5$ & 0.025 \\
\hline Estimated blood loss (ml) & $76.5 \pm 88.8$ & $79.2 \pm 81.9$ & 0.417 \\
\hline Surgery approach & & & 0.516 \\
\hline Laparoscopic & $158(69.0)$ & $155(67.6)$ & \\
\hline Open & $53(23.1)$ & $49(21.4)$ & \\
\hline Robotic & $18(7.9)$ & $25(10.9)$ & \\
\hline Post-operative hospital stay (days) & $8.3 \pm 5.5$ & $9.0 \pm 6.3$ & 0.183 \\
\hline Post-operative complications & $33(14.4)$ & $40(17.5)$ & 0.444 \\
\hline 30 days readmission & $1(0.4)$ & $1(0.4)$ & 1.000 \\
\hline Peri-CRT complications* & $64(27.9)$ & $62(27.1)$ & 0.917 \\
\hline Major & $7(3.1)$ & $1(0.4)$ & 0.068 \\
\hline Sphincter-saving procedure & $203(88.6)$ & $204(89.1)$ & 1.000 \\
\hline Lymph nodes retrieved & $12.4 \pm 7.7$ & $12.5 \pm 6.4$ & 0.832 \\
\hline Metastatic lymph nodes & $0.5 \pm 1.3$ & $0.5 \pm 1.5$ & 0.840 \\
\hline CRM involvement & $0(0)$ & $2(0.9)$ & 0.499 \\
\hline Pathological TNM stage & & & 0.957 \\
\hline 0 & $50(21.8)$ & $52(22.7)$ & \\
\hline | & $59(25.8)$ & $63(27.5)$ & \\
\hline$\|$ & $58(25.3)$ & $57(24.9)$ & \\
\hline III & $60(26.2)$ & $58(24.5)$ & \\
\hline IV & $2(0.9)$ & $1(0.4)$ & \\
\hline TRG grade & & & 0.026 \\
\hline 0 & $51(21.8)$ & $52(22.7)$ & 1.000 \\
\hline 1 & $84(36.7)$ & $61(26.6)$ & 0.027 \\
\hline 2 & 77 (33.6) & 105 (45.9) & 0.010 \\
\hline 3 & $17(7.4)$ & $11(4.8)$ & 0.330 \\
\hline Perineural invasion & $11(4.8)$ & 12 (5.2) & 1.000 \\
\hline Vascular invasion & $11(4.8)$ & $9(3.9)$ & 0.820 \\
\hline
\end{tabular}

*Some patients experienced more than one complication, and categorized as. NCRT, neoadjuvant chemoradiotherapy; NCRT-Cape, neoadjuvant chemoradiotherapy and additional capecitabine chemotherapy; CRM, circumferential resection margin; TRG, tumor regression grade. Bold values indicate of statistical significance.

\section{Definitions}

Tumor distance from the anal verge was estimated by digital rectal examination, pre-operative MRI evaluation, and intraoperative findings during the operation. Tumor response to NCRT was graded according to Rectal Cancer Tumor Regression Grade (TRG) method (24). pCR was defined as no viable tumor cells in the primary site or the lymph nodes. Postoperative morbidity was classified according to the ClavienDindo classification (25).

\section{Statistical Analysis}

To minimize group differences, we performed a 1:1 propensity score matching analysis by using $\mathrm{R}$ Version 3.5.1 (Vienna, Austria). Statistical analyses were performed using SPSS version 23.0 (SPSS INC., Chicago, IL, USA). Categorical variables were presented as numbers and compared using the chi-square test or Fisher's exact test as appropriate. Continuous variables were expressed as means \pm SD and analyzed using Student's $t$-test. The logistic regression model was used to identify independent predictors for $\mathrm{pCR}$, and a predictive nomogram was developed by the $\mathrm{R}$ project. To validate the results, patients were randomly divided into training $(n=420)$ and validation $(n=125)$ cohorts by using SPSS. The nomogram went through internal and external validation. $p<0.05$ was considered statistically significant.

\section{RESULTS}

\section{Patient Characteristics}

The baseline features of patients with LARC are presented in Table 1. Totally 545 patients with LARC were included. After propensity score matching, 229 patients receiving standard NCRT (NCRT group) and 229 patients treated with NCRT and additional 2 cycles of consolidation capecitabine chemotherapy (NCRT-Cape group) were matched. After matching, betweengroup baseline characteristics were well-balanced, such as age, gender, American Society of Anesthesiologists grade, interval time between NCRT and surgery, distance from the anal verge, clinical $\mathrm{T}$ and $\mathrm{N}$ stage.

\section{Perioperative Outcomes}

Surgical results are listed in Table 2. Estimated blood loss, surgical approach, and preserve organ rate were comparable between the two groups (Table 2). The operation time in the NCRT group was significantly lower than that of the NCRT-Cape group (213.2 \pm 67.4 vs. $227.9 \pm 70.5, p=0.025)$, compared to. Postoperative morbidity was similar between two groups (14.4 vs. $17.5 \%, p=0.444$ ). No group difference was observed in post-operative hospital stay and 30 days readmission ( $p$ $=0.183, p=1.000$, respectively). Complication severity was similar in the two groups. Similarly, no significant difference was observed in peri-NCRT complications between groups $(p=0.917)$. No re-operation was found in either group. Likewise, no perioperative mortality occurred in the two groups.

\section{Pathological Outcomes}

Adding consolidation capecitabine chemotherapy had no impact on lymph node retrieved and metastatic lymph nodes $(p=$ $0.832, p=0.840$, respectively). With regard to tumor response to NCRT, a lower proportion of good response (TRG1: 36.7 vs. $26.6 \%, p=0.028$ ) and a higher proportion of partial response (TRG2: 33.6 vs. $45.9 \%, p=0.010$ ) were noted in patients in the NCRT-Cape group. However, additional administration of 2 cycles of consolidation capecitabine chemotherapy did not increase pCR rate compared to standard NCRT group (21.8 vs. $22.7 \%, p=1.000$, Figure 2). Positive circumferential resection margin rates were comparable between both groups, and tumor size $(p=0.499, p=0.485)$. A pathological TNM stage was similar between two groups $(p=0.957)$. Similarly, perinerval and vascular invasion did not differ between two groups ( $p=1.000$, $p=0.820$, respectively). 


\section{Predictive Factors of pCR}

To identify risk factors for $\mathrm{pCR}$ in LARC, logistic regression analysis was performed in 545 patients (before propensity score matching). In univariate analysis, adding consolidation capecitabine chemotherapy ( $\mathrm{OR}=0.954, p=0.476)$ was not

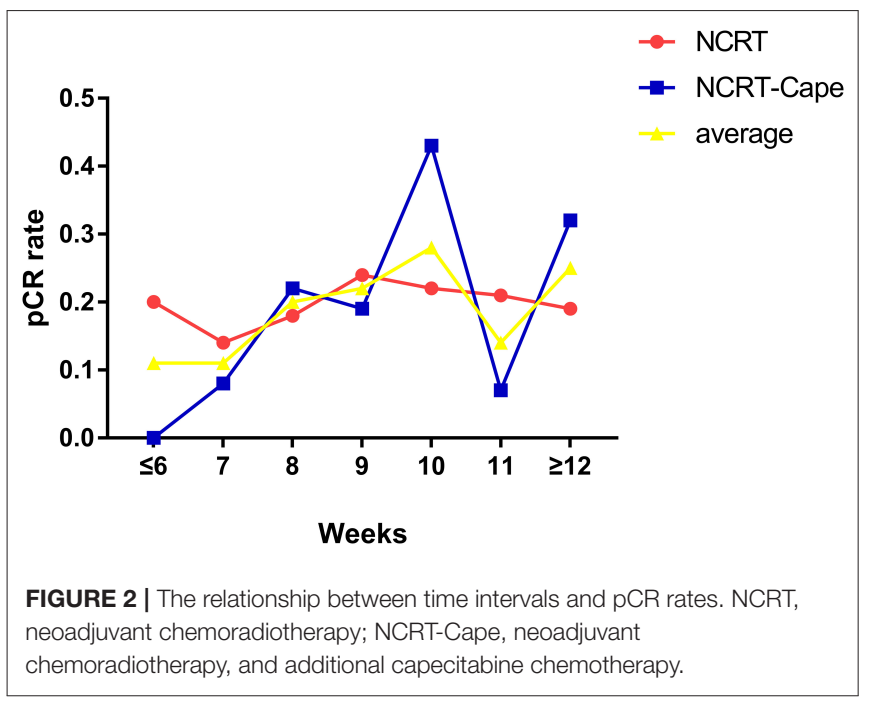

correlated with pCR in patients with LARC. Tumor size (OR $=0.428, p<0.001)$, interval time between NCRT and surgery $(\mathrm{OR}=1.141, p=0.036)$, pre-NCRT clinical $\mathrm{T}$ stage $(\mathrm{OR}=$ $0.641, p=0.027)$, pre-NCRT clinical $\mathrm{N}$ stage $(\mathrm{OR}=0.514, p=$ $0.031)$, lymph nodes harvested $(\mathrm{OR}=0.955, p=0.008)$, and postNCRT carcinoembryonic antigen $(\mathrm{CEA})(\mathrm{OR}=0.872, p=0.003)$ were significantly correlated with pCR in patients with LARC. On multivariate analysis, tumor size $(\mathrm{OR}=0.439, p<0.001)$, and interval time between NCRT and surgery $(\mathrm{OR}=1.241, p$ $=0.003)$, and post-NCRT CEA $(\mathrm{OR}=0.880, p=0.008)$ were significant risk factors for pCR in patients with LARC (Table 3 ).

\section{Nomogram for pCR}

Based on results from multivariate analysis, a predicting nomogram for pCR was developed, as demonstrated in Figure 3A. By summing up the score of each variable, a straight line could be drawn to obtain the predicted $\mathrm{pCR}$ rate. The C-index of the nomogram was 0.78 (95\% CI 0.73-0.83). The calibration curve (Figure 3B) showed good performance upon internal validation between the predicted and actual probability of pCR. Upon external validation, the C-index of the nomogram was 0.73 (95\% CI 0.63-0.83), and the calibration curve (Figure 3C) showed good accordance between predicted and observed probabilities of pCR.

TABLE 3 | Univariate and multivariate analysis of predictive factors for pCR in locally advanced rectal cancer patients $(n=545)$.

\begin{tabular}{|c|c|c|c|c|c|c|}
\hline \multirow[t]{2}{*}{ Variables } & \multicolumn{3}{|c|}{ Univariate analysis } & \multicolumn{3}{|c|}{ Multivariate analysis } \\
\hline & HR & $95 \% \mathrm{Cl}$ & $P$-Value & HR & $95 \% \mathrm{Cl}$ & $P$-Value \\
\hline Sex, male/female & 1.383 & $0.908-2.105$ & 0.926 & & & \\
\hline Age & 0.999 & $0.980-1.017$ & 0.886 & & & \\
\hline ASA & 0.916 & $0.594-1.411$ & 0.689 & & & \\
\hline Distance from the anal verge & 0.927 & $0.850-1.011$ & 0.088 & & & \\
\hline Tumor size & 0.428 & $0.334-0.548$ & $<0.001$ & 0.439 & $0.338-0.570$ & $<0.001$ \\
\hline \multicolumn{7}{|l|}{ Surgery approach } \\
\hline Laparoscopic & Reference & Reference & 0.724 & & & \\
\hline Open & 1.141 & $0.530-2.455$ & 0.736 & & & \\
\hline Robotic & 1.348 & $0.582-3.122$ & 0.486 & & & \\
\hline Sphincter-Saving procedure & 0.584 & $0.279-1.221$ & 0.153 & & & \\
\hline Operative time (min) & 0.998 & 0.995-1.002 & 0.337 & & & \\
\hline Estimated blood loss (ml) & 0.998 & $0.995-1.001$ & 0.284 & & & \\
\hline Time interval between NCRT and surgery & 1.141 & $1.009-1.291$ & 0.036 & 1.241 & $1.074-1.434$ & 0.003 \\
\hline Pre-NCRT cT stage & 0.641 & $0.432-0.950$ & 0.027 & 0.710 & $0.460-1.096$ & 0.123 \\
\hline Pre-NCRT cN stage & 0.514 & $0.281-0.941$ & 0.031 & 0.707 & $0.355-1.407$ & 0.323 \\
\hline Post-Operative hospital stay & 0.969 & $0.927-1.014$ & 0.175 & & & \\
\hline Lymph nodes harvested & 0.955 & $0.924-0.988$ & 0.008 & 0.985 & $0.948-1.022$ & 0.420 \\
\hline Post-NCRT CEA level & 0.872 & $0.795-0.956$ & 0.003 & 0.880 & $0.802-0.967$ & 0.008 \\
\hline Post-NCRT CA19-9 level & 0.993 & $0.979-1.007$ & 0.309 & & & \\
\hline Post-Operative complications & 0.821 & $0.456-1.475$ & 0.666 & & & \\
\hline Plus capecitabine & 0.954 & $0.632-1.440$ & 0.822 & & & \\
\hline Reduce the dose & 1.237 & $0.246-6.210$ & 0.796 & & & \\
\hline NCRT complications & 1.139 & $0.721-1.800$ & 0.577 & & & \\
\hline
\end{tabular}

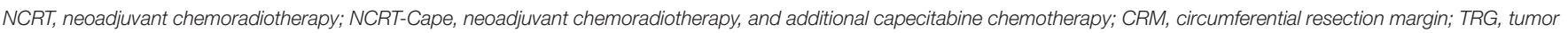
regression grade, HR, hazard ratio; $\mathrm{Cl}$, confidential interval. Bold values indicate of statistical significance. 


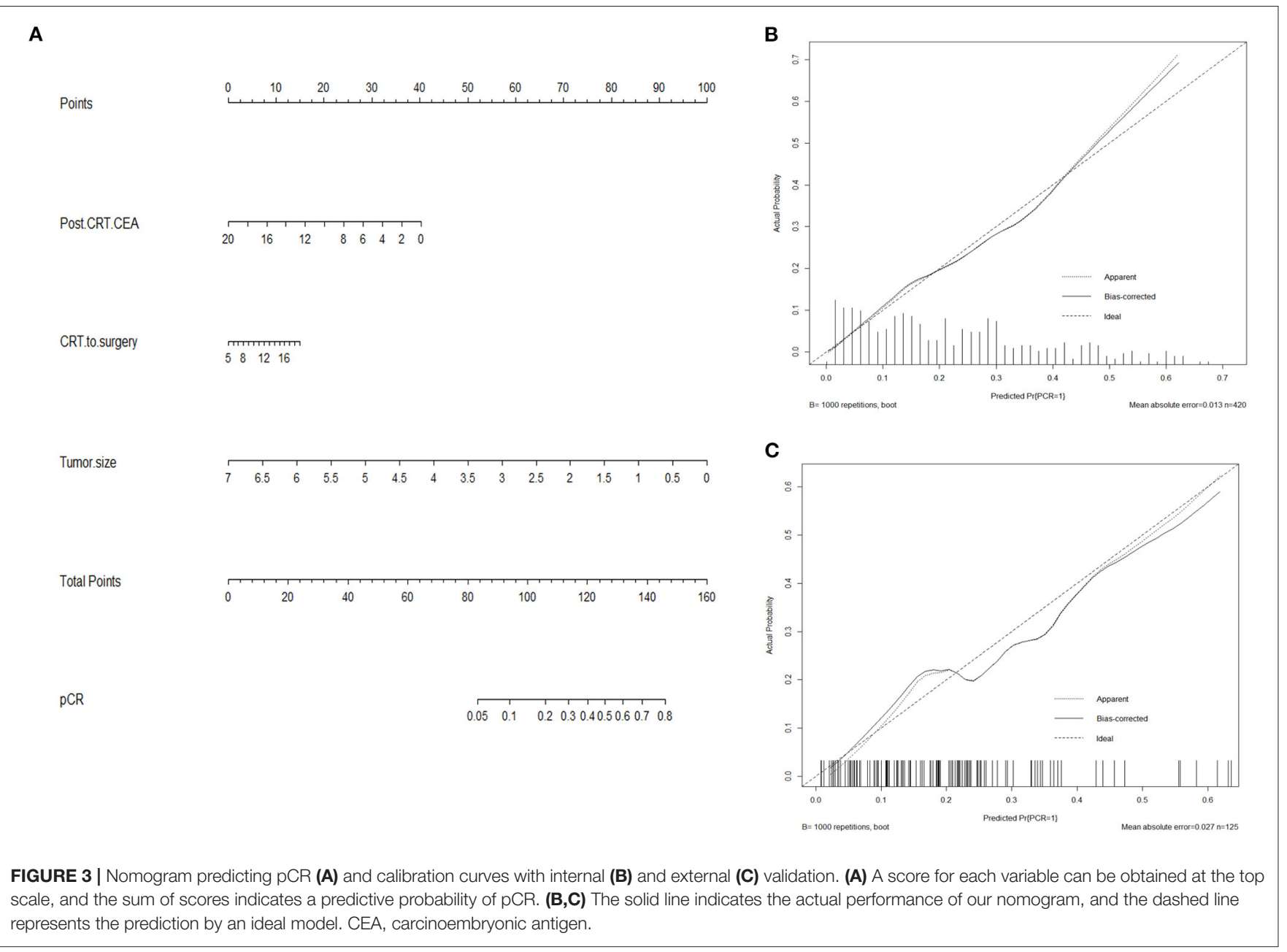

\section{DISCUSSION}

A great effort has been made to maximize tumor response to NCRT, in which pCR is selected as a surrogate endpoint. Herein, we investigated the efficacy of adding consolidation capecitabine chemotherapy without changing the CRT-to-surgery interval for patients with LARC. The result demonstrated that adding 2 cycles of consolidation capecitabine to NCRT had no impact on the short-term perioperative outcome, and did not result in an increase in pCR rates. Additionally, by incorporating postNCRT significant predictive factors in Logistic analysis, we built a nomogram for pCR that might assist in decision-making about organ-preserving strategies.

Fluoropyrimidine-based (5-FU or capecitabine) pre-operative chemoradiotherapy is the standard care for LARC. Incorporation of oxaliplatin to fluoropyrimidine-based CRT has been shown to acquire equivalent oncological outcomes but increase toxicities (18-22). Accumulating evidence has proposed capecitabine to be an alternative to 5-FU, the efficacy of XELOX (capecitabine and oxaliplatin) was comparable with that of the FOLFOX4 regimen (5-FU/folinic acid plus oxaliplatin) (26). In addition, using oral capecitabine instead of infusional 5-FU during NCRT has been shown to be correlated with improved tumor response and lower toxicity, and a comparable pCR rate is compared to preoperative 5-FU-based NCRT (27-29). It has been demonstrated that nCRT with capecitabine is safe and well-tolerated in the ACCORD/PRODIGD 2 phase III trial (30).

To improve tumor response without increasing perioperative complications, we added 2 cycles of consolidation capecitabine chemotherapy in the waiting period to the standard NCRT regimen. Our preliminary results demonstrated that additional two-cycle consolidation capecitabine did not increase the incidence of peri-CRT complications, such as hand-foot syndrome, fatigue, and diarrhea, suggesting this regimen might be well-tolerated.

Meanwhile, we used propensity score matching to reduce selection bias; between-group baseline characteristics were well-balanced after matching. No negative effect on surgical outcome was observed when patients were administered twocycle consolidation capecitabine to NCRT. Regarding surgical morbidity, the severity of post-operative complications and post-operative hospital stay were found comparable between the two groups. No reoperation and perioperative mortality occurred in the two groups. Together, these results indicated the 
safety of adding 2 cycles of consolidation capecitabine to the NCRT regimen.

To further explore the efficacy of adding consolidation capecitabine to NCRT on tumor response, we compared the pCR rates between the two groups. The addition of consolidation capecitabine to NCRT did not significantly improve pCR rate. The absolute ypCR rate difference between groups was very small and not statistically significant. Similar results were found in a recent phase II OIGIT-01 Trial (31). We suggested that radiosensitization by using capecitabine might have already maximized tumor responses to NCRT, leaving little room for improvement with the addition of two-cycle consolidation capecitabine chemotherapy.

The optimal timing of surgery after NCRT is still controversial (32). Surgery beyond 8 weeks after completion of radiotherapy might increase pelvic fibrosis and rectal edema, leading to intraoperative technical difficulties and increased surgical complications. On the other hand, the pCR rate might increase by prolongation of the NCRT-to-surgery interval (17,33-36). Herein, we analyzed the relationship between pCR and NCRT-to-surgery interval. The result revealed that longer interval time correlated with increased PCR rate. Results from Logistic regression demonstrated that interval time was a significant risk factor for $\mathrm{pCR}$ than the consolidation capecitabine chemotherapy.

Additionally, after adjustment for confounding factors, tumor size, and post-NCRT CEA level were significant risk factors for $\mathrm{pCR}$ in patients with LARC. To facilitate the decisionmaking regarding organ-preserving strategies, we developed a nomogram predicting $\mathrm{pCR}$. This nomogram has a reliable $\mathrm{C}$ index on internal and external validation. The incorporation of specific molecular and genetic markers into the predicting nomogram would enhance the performance of the model. Nowadays, delivery of "total neoadjuvant therapy" (TNT) strategies is becoming increasingly popular to improve the pCR rates (37), allowing a group of patients to benefit from fulldose adjuvant chemotherapy and finally a less-invasive organ preservation strategy (38-41). However, the definite role of TNT strategies is still unveiled.

There are several limitations that warrant discussion. First, this study was subjected to selection bias owing to its retrospective nature. To minimize selection bias between groups, we performed propensity score analysis. Second, our predictive model was based on a single-center retrospective analysis. It requires further external validation in a large population from multiple institutions. Another limitation was that this study

\section{REFERENCES}

1. Heald RJ, Husband EM, Ryall RD. The mesorectum in rectal cancer surgery-the clue to pelvic recurrence. $\mathrm{Br} J$ Surg. (1982) 69:613-6. doi: 10.1002/bjs.1800691019

2. Sauer R, Becker H, Hohenberger W, Rödel C, Wittekind C, Fietkau $\mathrm{R}$, et al. Preoperative versus postoperative chemoradiotherapy for rectal cancer. N Engl J Med. (2004) 351:1731-40. doi: 10.1056/NEJMoa0 40694 focused on the pCR rate, a surrogate marker of oncological outcomes. Further studies focused on the long-term oncological outcomes are needed to further confirm the results of our study. Nevertheless, our study adds to the understanding of the efficacy of adding capecitabine to standard NCRT.

Our study suggested that additional studies in a large-scale population are needed to confirm these results.

\section{DATA AVAILABILITY STATEMENT}

The original contributions presented in the study are included in the article/Supplementary Material, further inquiries can be directed to the corresponding author/s.

\section{ETHICS STATEMENT}

Studies relative to humans in this article were approved by the Ethics Committee of The Fujian Medical University Union Hospital (2013051). The patients/participants provided their written informed consent to participate in this study.

\section{AUTHOR CONTRIBUTIONS}

YFF, CMS, FD, WJZ, GXG, and XL designed the experiments, performed the experiments, analyzed the data, and wrote the article. All authors contributed to the article and approved the submitted version.

\section{FUNDING}

This study was supported by the National Foundation of China (No. 82172800), Science Foundation of the Fujian Province (No. 2019J0105), Special Financial Foundation of Fujian Provincial (No. 2015-1297 and 2020B1050), the Startup Fund for Scientific Research, Fujian Medical University (2017XQ1029 and 2018QH2027), and the Professor Development Foundation of Fujian Medical University (No. JS11006). Talent programs granted from The First Affiliated Hospital of Fujian Medical University (YJRC3600). Joint Funds for the Innovation of Science and Technology, Fujian Province (2020Y9125).

\section{SUPPLEMENTARY MATERIAL}

The Supplementary Material for this article can be found online at: https://www.frontiersin.org/articles/10.3389/fsurg. 2021.770767/full\#supplementary-material 
5. Park IJ, You YN, Agarwal A, Skibber JM, Rodriguez-Bigas MA, Eng C, et al. Neoadjuvant treatment response as an early response indicator for patients with rectal cancer. J Clin Oncol. (2012) 30:1770-6. doi: 10.1200/JCO.2011.39.7901

6. Maas M, Nelemans PJ, Valentini V, Das P, Rödel C, Kuo LJ, et al. Longterm outcome in patients with a pathological complete response after chemoradiation for rectal cancer: a pooled analysis of individual patient data. Lancet Oncol. (2010) 11:835-44. doi: 10.1016/S1470-2045(10)70172-8

7. Roh MS, Colangelo LH, O'Connell MJ, Yothers G, Deutsch M, Allegra CJ, et al. Preoperative multimodality therapy improves disease-free survival in patients with carcinoma of the rectum: NSABP R-03. J Clin Oncol. (2009) 27:5124-30. doi: 10.1200/JCO.2009.22.0467

8. São Julião GP, Habr-Gama A, Vailati BB, Aguilar PB, Sabbaga J, Araújo S, et al. Is neoadjuvant chemoradiation with dose-escalation and consolidation chemotherapy sufficient to increase surgery-free and distant metastases-free survival in baseline cT3 rectal cancer. Eur J Surg Oncol. (2018) 44:939. doi: 10.1016/j.ejso.2017.11.010

9. Wiltshire KL, Ward IG, Swallow C, Oza AM, Cummings B, Pond GR, et al. Preoperative radiation with concurrent chemotherapy for resectable rectal cancer: effect of dose escalation on pathologic complete response, local recurrence-free survival, disease-free survival, and overall survival. Int J Radiat Oncol Biol Phys. (2006) 64:709-16. doi: 10.1016/j.ijrobp.2005.08.012

10. Fernández-Martos C, Pericay C, Aparicio J, Salud A, Safont M, Massuti B, et al. Phase II, randomized study of concomitant chemoradiotherapy followed by surgery and adjuvant capecitabine plus oxaliplatin (CAPOX) compared with induction CAPOX followed by concomitant chemoradiotherapy and surgery in magnetic resonance imaging-defined, locally advanced rectal cancer: grupo cancer de recto 3 study. J Clin Oncol. (2010) 28:85965. doi: 10.1200/JCO.2009.25.8541

11. Fernandez-Martos C, Garcia-Albeniz X, Pericay C, Maurel J, Aparicio J, Montagut $\mathrm{C}$, et al. Chemoradiation, surgery and adjuvant chemotherapy versus induction chemotherapy followed by chemoradiation and surgery: long-term results of the Spanish GCR-3 phase II randomized trial ${ }^{\dagger}$. Ann Oncol. (2015) 26:1722-8. doi: 10.1093/annonc/mdv223

12. Myerson RJ, Tan B, Hunt S, Olsen J, Birnbaum E, Fleshman J, et al. Five fractions of radiation therapy followed by 4 cycles of FOLFOX chemotherapy as preoperative treatment for rectal cancer. Int J Radiat Oncol Biol Phys. (2014) 88:829-36. doi: 10.1016/j.ijrobp.2013.12.028

13. Markovina S, Youssef F, Roy A, Aggarwal S, Khwaja S, DeWees T, et al. Improved metastasis- and disease-free survival with preoperative sequential short-course radiation therapy and FOLFOX chemotherapy for rectal cancer compared with neoadjuvant long-course chemoradiotherapy: results of a matched pair analysis. Int J Radiat Oncol Biol Phys. (2017) 99:41726. doi: 10.1016/j.ijrobp.2017.05.048

14. Abboud H, Petrak A, Mealy M, Sasidharan S, Siddique L, Levy M. Treatment of acute relapses in neuromyelitis optica: steroids alone versus steroids plus plasma exchange. Mult Scler. (2016) 22:185-92. doi: 10.1177/1352458515581438

15. Cercek A, Goodman KA, Hajj C, Weisberger E, Segal NH, Reidy-Lagunes DL, et al. Neoadjuvant chemotherapy first, followed by chemoradiation and then surgery, in the management of locally advanced rectal cancer. J Natl Compr Canc Netw. (2014) 12:513-9. doi: 10.6004/jnccn.2014.0056

16. Bujko K, Wyrwicz L, Rutkowski A, Malinowska M, Pietrzak L, Kryński J, et al. Long-course oxaliplatin-based preoperative chemoradiation versus $5 \times 5 \mathrm{~Gy}$ and consolidation chemotherapy for cT4 or fixed cT3 rectal cancer: results of a randomized phase III study. Ann Oncol. (2016) 27:83442. doi: 10.1093/annonc/mdw198.20

17. Du D, Su Z, Wang D, Liu W, Wei Z. Optimal interval to surgery after neoadjuvant chemoradiotherapy in rectal cancer: a systematic review and meta-analysis. Clin Colorectal Cancer. (2018) 17:13-24. doi: 10.1016/j.clcc.2017.10.012

18. De Felice F, Benevento I, Magnante AL, Musio D, Bulzonetti N, Caiazzo $\mathrm{R}$, et al. Clinical benefit of adding oxaliplatin to standard neoadjuvant chemoradiotherapy in locally advanced rectal cancer: a meta-analysis : oxaliplatin in neoadjuvant treatment for rectal cancer. BMC Cancer. (2017) 17:325. doi: 10.1186/s12885-017-3323-4

19. Gérard JP, Azria D, Gourgou-Bourgade S, Martel-Laffay I, Hennequin C, Etienne PL, et al. Comparison of two neoadjuvant chemoradiotherapy regimens for locally advanced rectal cancer: results of the phase III trial ACCORD 12/0405-Prodige 2. J Clin Oncol. (2010) 28:1638-44. doi: 10.1200/JCO.2009.25.8376

20. Rödel C, Graeven U, Fietkau R, Hohenberger W, Hothorn T, Arnold D, et al. Oxaliplatin added to fluorouracil-based preoperative chemoradiotherapy and postoperative chemotherapy of locally advanced rectal cancer (the German CAO/ARO/AIO-04 study): final results of the multicentre, open-label, randomised, phase 3 trial. Lancet Oncol. (2015) 16:97989. doi: 10.1016/S1470-2045(15)00159-X

21. Aschele C, Cionini L, Lonardi S, Pinto C, Cordio S, Rosati G, et al. Primary tumor response to preoperative chemoradiation with or without oxaliplatin in locally advanced rectal cancer: pathologic results of the STAR-01 randomized phase III trial. J Clin Oncol. (2011) 29:2773-80. doi: 10.1200/JCO.2010.34.4911

22. Benson AB, Venook AP, Al-Hawary MM, Cederquist L, Chen YJ, Ciombor KK, et al. Rectal cancer, version 2.2018, NCCN clinical practice guidelines in oncology. J Natl Compr Canc Netw. (2018) 16:874901. doi: 10.6004/jncen.2018.0061

23. Zou XC, Wang QW, Zhang JM. Comparison of 5-FU-based and capecitabine-based neoadjuvant chemoradiotherapy in patients with rectal cancer: a meta-analysis. Clin Colorectal Cancer. (2017) 16:e123-123e139. doi: 10.1016/j.clcc.2017.01.009

24. Wheeler JM, Warren BF, Mortensen NJ, Ekanyaka N, Kulacoglu H, Jones AC, et al. Quantification of histologic regression of rectal cancer after irradiation: a proposal for a modified staging system. Dis Colon Rectum. (2002) 45:10516. doi: 10.1007/s10350-004-6359-x

25. Dindo D, Demartines N, Clavien PA. Classification of surgical complications: a new proposal with evaluation in a cohort of 6336 patients and results of a survey. Ann Surg. (2004) 240:205-13. doi: 10.1097/01.sla.0000133083.54934.ae

26. Cassidy J, Saltz L, Twelves C, Van Cutsem E, Hoff P, Kang Y, et al. Efficacy of capecitabine versus 5-fluorouracil in colorectal and gastric cancers: a metaanalysis of individual data from 6171 patients. Ann Oncol. (2011) 22:26049. doi: 10.1093/annonc/mdr031

27. Fernández-Martos C, Nogué M, Cejas P, Moreno-García V, Machancoses AH, Feliu J. The role of capecitabine in locally advanced rectal cancer treatment: an update. Drugs. (2012) 72:1057-73. doi: 10.2165/11633870-000000000-00000

28. Hofheinz RD, Wenz F, Post S, Matzdorff A, Laechelt S, Hartmann JT, et al. Chemoradiotherapy with capecitabine versus fluorouracil for locally advanced rectal cancer: a randomised, multicentre, non-inferiority, phase 3 trial. Lancet Oncol. (2012) 13:579-88. doi: 10.1016/S1470-2045(12)70116-X

29. Chan AK, Wong AO, Jenken DA. Preoperative capecitabine and pelvic radiation in locally advanced rectal cancer-is it equivalent to 5-FU infusion plus leucovorin and radiotherapy. Int J Radiat Oncol Biol Phys. (2010) 76:1413-9. doi: 10.1016/j.ijrobp.2009.03.048

30. François E, Azria D, Gourgou-Bourgade S, Jarlier M, Martel-Laffay I, Hennequin C, et al. Results in the elderly with locally advanced rectal cancer from the ACCOR12/PRODIGE 2 phase III trial: tolerance and efficacy. Radiother Oncol. (2014) 110:144-9. doi: 10.1016/j.radonc.2013.10.019

31. Golo D, But-Hadzic J, Anderluh F, Brecelj E, Edhemovic I, Jeromen A, et al. Induction chemotherapy, chemoradiotherapy and consolidation chemotherapy in preoperative treatment of rectal cancer - longterm results of phase II OIGIT-01 trial. Radiol Oncol. (2018) 52:267-74. doi: 10.2478/raon-2018-0028

32. Glimelius B. Optimal time intervals between pre-operative radiotherapy or chemoradiotherapy and surgery in rectal cancer. Front Oncol. (2014) 4:50. doi: $10.3389 /$ fonc. 2014.00050

33. Figueiredo N, Panteleimonitis S, Popeskou S, Cunha JF, Qureshi T, Beets GL, et al. Delaying surgery after neoadjuvant chemoradiotherapy in rectal cancer has no influence in surgical approach or short-term clinical outcomes. Eur J Surg Oncol. (2018) 44:484-9. doi: 10.1016/j.ejso.2018.01.088

34. Garcia-Aguilar J, Smith DD, Avila K, Bergsland EK, Chu P, Krieg RM. Optimal timing of surgery after chemoradiation for advanced rectal cancer: preliminary results of a multicenter, nonrandomized phase II prospective trial. Ann Surg. (2011) 254:97-102. doi: 10.1097/SLA.0b013e3182196elf

35. Rombouts A, Hugen N, Elferink M, Nagtegaal ID, de Wilt J. Treatment interval between neoadjuvant chemoradiotherapy and surgery in rectal cancer patients: a population-based study. Ann Surg Oncol. (2016) 23:3593601. doi: 10.1245/s10434-016-5294-0 
36. Petrelli F, Sgroi G, Sarti E, Barni S. Increasing the interval between neoadjuvant chemoradiotherapy and surgery in rectal cancer: a meta-analysis of published Studies. Ann Surg. (2016) 263:458-64. doi: 10.1097/SLA.00000000000 00368

37. Garcia-Aguilar J, Chow OS, Smith DD, Marcet JE, Cataldo PA, Varma MG, et al. Effect of adding mFOLFOX6 after neoadjuvant chemoradiation in locally advanced rectal cancer: a multicentre, phase 2 trial. Lancet Oncol. (2015) 16:957-66. doi: 10.1016/S1470-2045(15)00004-2

38. Probst CP, Becerra AZ, Aquina CT, Tejani MA, Wexner SD, Garcia-Aguilar J, et al. Extended intervals after neoadjuvant therapy in locally advanced rectal cancer: the key to improved tumor response and potential organ preservation. J Am Coll Surg. (2015) 221:430-40. doi: 10.1016/j.jamcollsurg.2015. 04.010

39. Nilsson PJ, van Etten B, Hospers GA, Påhlman L, van de Velde CJ, Beets-Tan $\mathrm{RG}$, et al. Short-course radiotherapy followed by neo-adjuvant chemotherapy in locally advanced rectal cancer-the RAPIDO trial. BMC Cancer. (2013) 13:279. doi: 10.1186/1471-2407-13-279

40. Martens MH, Maas M, Heijnen LA, Lambregts DM, Leijtens JW, Stassen LP, et al. Long-term outcome of an organ preservation program after neoadjuvant treatment for rectal cancer. J Natl Cancer Inst. (2016) 108:djw171. doi: 10.1093/jnci/djw171
41. Beets GL, Figueiredo NF, Beets-Tan RG. Management of rectal cancer without radical resection. Annu Rev Med. (2017) 68:169-82. doi: 10.1146/annurev-med-062915-021419

Conflict of Interest: The authors declare that the research was conducted in the absence of any commercial or financial relationships that could be construed as a potential conflict of interest.

Publisher's Note: All claims expressed in this article are solely those of the authors and do not necessarily represent those of their affiliated organizations, or those of the publisher, the editors and the reviewers. Any product that may be evaluated in this article, or claim that may be made by its manufacturer, is not guaranteed or endorsed by the publisher.

Copyright (c) 2022 Fang, Sheng, Ding, Zhao, Guan and Liu. This is an open-access article distributed under the terms of the Creative Commons Attribution License (CC $B Y)$. The use, distribution or reproduction in other forums is permitted, provided the original author(s) and the copyright owner(s) are credited and that the original publication in this journal is cited, in accordance with accepted academic practice. No use, distribution or reproduction is permitted which does not comply with these terms. 